\title{
Thermally excited Trivelpiece-Gould modes as a pure electron plasma temperature diagnostic ${ }^{\text {a) }}$
}

\author{
F. Anderegg, ${ }^{\text {b) }}$ N. Shiga, D. H. E. Dubin, and C. F. Driscoll \\ Institute for Pure and Applied Physical Sciences and Department of Physics, \\ University of California at San Diego, La Jolla, California 92093 \\ R. W. Gould \\ California Institute of Technology, Mail Stop 128-95, Pasadena, California 91103
}

(Received 11 November 2002; accepted 19 December 2002)

\begin{abstract}
Thermally excited plasma modes are observed in trapped, near-thermal-equilibrium pure electron plasmas over a temperature range of $0.05<k T<5 \mathrm{eV}$. The modes are excited and damped by thermal fluctuations in both the plasma and the receiver electronics. The thermal emission spectra together with a plasma-antenna coupling coefficient calibration uniquely determine the plasma (and load) temperature. This calibration is obtained from the mode spectra themselves when the receiver-generated noise absorption is measurable; or from separate wave reflection/absorption measurements; or from kinetic theory. This nondestructive temperature diagnostic agrees well with standard diagnostics, and may be useful for expensive species such as antimatter.
\end{abstract}

(c) 2003 American Institute of Physics. [DOI: 10.1063/1.1559973]

\section{INTRODUCTION}

Un-neutralized plasmas are unique in that they can be trapped in a rotating thermal equilibrium state by static electric and magnetic fields. Steady-state confinement of $N$ $=10^{3}-10^{9}$ electrons, ions, or antimatter particles ${ }^{1,2}$ is routinely used in plasma experiments, atomic physics, ${ }^{3}$ and spectroscopy. ${ }^{4}$ The thermal equilibrium characteristics become most evident with the formation of Coulomb crystals ${ }^{5}$ when pure ion plasmas are cooled to the liquid and solid regimes at sub-Kelvin temperatures. The higher temperature plasma regime studied here is also well-described by nearequilibrium statistical mechanics, ${ }^{6}$ with kinetic theories of waves and transport coefficients ${ }^{7}$ amenable to experimental tests.

These stable thermal equilibrium plasmas exhibit fluctuating electric fields due to the random motions of the particles. The weakly damped plasma waves represent normal modes of the isolated plasma, with expected average electrostatic potential energy of $\frac{1}{2} k T_{p}$ where $T_{p}$ is the plasma temperature. These waves are excited by particles as they move randomly in the plasma; and if a receiver is connected to the plasma, the waves will also be excited by uncorrelated thermal fluctuations in the receiver circuit. Conversely, the waves are absorbed (e.g., Landau-damped) by the particles and by the real part of the load impedance. In traps with finite length and radius the modes are discrete, and the Fourier spectra of the electric fields exhibit well-separated peaks at the discrete Trivelpiece-Gould (TG) standing mode frequencies. ${ }^{8}$

Somewhat simpler center of mass modes are commonly observed in the "single particle regime" with highly tuned resonant circuits in hyperbolic traps. ${ }^{9}$ The center of mass

\footnotetext{
${ }^{a)}$ Paper QI2 2, Bull. Am. Phys. Soc. 47, 249 (2002).

${ }^{b)}$ Invited speaker. Electronic mail: fanderegg @ucsd.edu
}

mode is a well-established diagnostic for particle number; but these oscillations are only weakly coupled to the random particle motion through the anharmonicity of the trap, and little temperature information can be obtained. Thermal excitation of the drift (diocotron) modes at lower frequencies is more difficult to measure; whereas thermal cyclotron modes at higher frequencies are readily observed in warm non-neutral ${ }^{10}$ and hot fusion plasmas; ${ }^{11}$ upper hybrid modes have also been used as a thermal diagnostic. ${ }^{12}$ In space plasmas, thermal noise is also used as a diagnostic, ${ }^{13}$ there the absence of boundary conditions significantly changes the impedance of a mode near a resonance. In the crystallized regime, suprathermal equipartition of mode energy has recently been observed in dusty plasmas. ${ }^{14}$ In mirrors of a laser resonator, ${ }^{15}$ thermally excited vibrations are carefully analyzed assuming that each mode has energy $k T$.

Historically, the ionospheric microwave back scattering is probably one of the first observations of radiation scattering by a collective effect, i.e., not by individual particles. ${ }^{16,17}$ Similarly, the signals detected in the present experiment are the result of collective plasma modes and not the result of uncorrelated charged particles moving randomly.

In this paper, the spectrum of thermally excited TG standing modes is measured in pure electron plasmas over a temperature range of $0.05<k T_{p}<5 \mathrm{eV}$, using a roomtemperature receiver (with an effective temperature $\sim 0.05$ $\mathrm{eV}-0.1 \mathrm{eV})$. We consider the azimuthally symmetric $\left(m_{\theta}\right.$ $=0$ ) modes only. The received spectrum consists of a Lorentzian due to the plasma mode, plus an uncorrelated broad spectrum due to the receiver (load) noise. Each of these components is "filtered" by the load impedance $Z_{\ell}$ and the mode impedance $Z_{m}$. When the receiver impedance is non-negligible compared to the mode impedance, a nonLorentzian spectrum occurs: resonant absorption of the re- 

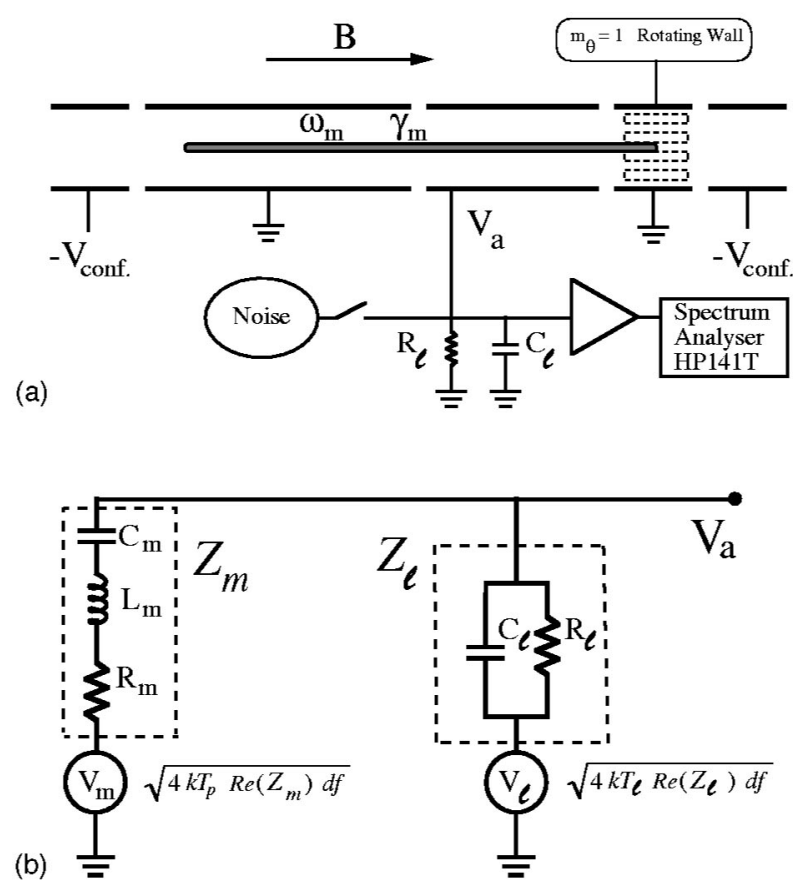

FIG. 1. (a) Schematic diagram of a cylindrical Penning-Malmberg trap with the receiving circuit. (b) Electrical circuit analogue to the plasma mode and receiver.

ceiver noise by the plasma mode creates a characteristic "dip and peak" in the noise spectrum.

By Nyquist's theorem, ${ }^{10}$ thermal plasmas generate noise proportional to $k T_{p}$ times the real part of the plasma mode impedance, $Z_{m}^{\mathrm{Re}}$. Conversely, the load-generated noise is proportional to the real part of the load impedance $Z_{\ell}^{\mathrm{Re}}$ times $k T_{\ell}$. The area of the received spectral peak is proportional to the average mode energy $W_{m}$, normalized by the geometrical antenna plasma coupling. The average mode energy is in equilibrium with both the plasma temperature and the load temperature; more precisely, we will see that $W_{m}$ is coupled to $k T_{p}$ at the mode damping rate $\gamma_{m}$ and is coupled to $k T_{\ell}$ at a load damping rate $\gamma_{\ell}$. The plasma impedance $Z_{m}$ can be obtained directly from the received noise spectra when the receiver impedance and noise are significant; or it can be obtained by a separate wave reflection/absorption measurement; or it can be calculated from a kinetic theory of random test particles. Overall, the technique allows a rapid nondestructive diagnostic of the plasma temperature with $\pm 25 \%$ accuracy.

\section{EXPERIMENTAL APPARATUSES}

Fluctuation measurements were obtained from pure electron plasmas contained in two similar Penning-Malmberg traps ("IV" and "EV") shown schematically in Fig. 1(a). These two traps differ mainly in plasma diameter and magnetic field strength. The IV trap consists of a series of hollow conducting cylinders of radius $r_{w}=2.86 \mathrm{~cm}$ contained in ultrahigh vacuum at $P \approx 10^{-10}$ Torr with a uniform axial magnetic field of $B=30 \mathrm{kG}$. Electrons are injected from a hot tungsten filament, and contained axially by voltages $V_{\text {conf }}$ $\approx-200 \mathrm{~V}$ on end electrodes. Typical plasmas have $N \approx 10^{9}$ electrons in a column length $L_{p} \approx 41 \mathrm{~cm}$, with a plasma radius $r_{p} \approx 0.2 \mathrm{~cm}$ and a central density $n_{0} \approx 10^{7} \mathrm{~cm}^{-3}$. (For $\mathrm{EV}$, the parameters are $B=0.375 \mathrm{kG}, r_{p}=1.7 \mathrm{~cm}, r_{w}=3.8$ $\mathrm{cm}$, and $L_{p}=24 \mathrm{~cm}$.)

The plasma density profile $n(r)$ and the temperature $T_{p}$ are obtained by dumping the plasma axially and measuring the total charge passing through a hole in a scanning collimator plate. Both measurements require shot-to-shot reproducibility of the injected plasma, and we typically observe variability $\delta n / n \leqslant 1 \%$. On IV, a weak "rotating wall" perturbation at $f_{\mathrm{RW}} \sim 0.5 \mathrm{MHz}$ is used to obtain steady-state confinement of the electron column. ${ }^{18}$ The EV plasmas expand radially towards the wall with a characteristic "mobility" time of $\tau_{m} \approx 100 \mathrm{~s}$, so the electrons are repetitively injected and dumped. The spectrum analyzer scans analyzed here typically require about $0.5 \mathrm{~s}$ to complete.

The parallel temperature $T_{\|}$of the electrons can be measured by slowly lowering the confinement voltage and measuring the escaping charge. ${ }^{19}$ On EV, the perpendicular temperature $T_{\perp}$ can also be measured using a "magnetic beach" analyzer. In general, we find $T_{\|} \approx T_{\perp} \equiv T_{p}$, since the electron-electron collision rate $\nu_{\perp \|} \approx 100 \mathrm{~s}^{-1}$ is relatively rapid. ${ }^{20} \mathrm{On} \mathrm{EV}$, the electrons equilibrate to $k T_{p} \approx 1 \mathrm{eV}$ soon after injection, whereas the electrons in IV cool to $k T_{p}$ $\approx 0.05 \mathrm{eV}$ due to cyclotron radiation. To control the temperature, we apply auxiliary "wiggle" heating by modulating one electrode voltage at a frequency $f_{h}=0.8-1.0 \mathrm{MHz}$, where $f_{h}$ is adjusted so that all harmonics are distinct from the TG mode of interest. On the EV apparatus, a "heating burst" is applied before the measurement; in contrast, on the IV apparatus, the heating is applied continuously to balance the cooling due to the cyclotron radiation.

\section{MODE SPECTRA}

We perform plasma wave transmission experiments by applying an rf voltage of amplitude $V_{\text {exc }}$ to a cylindrical electrode at one end of the column. $V_{\text {exc }}$ excites density perturbations $\delta n$ in the column which propagate and induce the measured voltages $V_{a}$ on a distant receiving cylindrical antenna with finite load impedance. Here, the wave transmission cylinder has length $L_{\mathrm{exc}}=5.8 \mathrm{~cm}$, and the cylinder used as a reception antenna has $L_{a}=11.7 \mathrm{~cm}$. The load impedance on IV (or EV) is $R_{\ell}=750 \Omega$ (or $50 \Omega$ ) in parallel with $C_{\ell}=440 \mathrm{pF}$ (or $165 \mathrm{pF}$ ).

Figures 2(a) and 2(b) show the spectrum of standing $m_{\theta}=0$ Trivelpiece-Gould modes excited by wall excitations of $V_{\text {exc }}=-80 \mathrm{dbm}(22 \mu \mathrm{V})$ and $-100 \mathrm{dBm}(2.2 \mu \mathrm{V})$ at frequencies $f=0.01-10 \mathrm{MHz}$. The frequencies of the Trivelpiece-Gould mode can be approximated as

$$
f \approx f_{p}\left(\frac{r_{p}}{r_{w}}\right)\left(\frac{r_{w} \pi m_{z}}{L_{p}}\right)\left[\frac{1}{2} \ln \frac{r_{w}}{r_{p}}\right]^{1 / 2}\left[1+\frac{3}{2}\left(\frac{\bar{v}}{v_{\phi}}\right)^{2}\right] .
$$

The wave frequencies scale with the plasma frequency $f_{p}$ $\equiv 28 \mathrm{MHz}\left(n / 10^{7} \mathrm{~cm}^{-3}\right)^{1 / 2}$, reduced by the fill ratio $r_{p} / r_{w}$ and by the trap radius compared to the axial wavelength. ${ }^{21}$ The axial wave number is given by $k_{z}=\pi m_{z} / L_{p}$, where axial mode number is $m_{z}=1,2, \ldots, 5$. We have also included thermal corrections, which depend on the ratio of $\bar{v}$ 


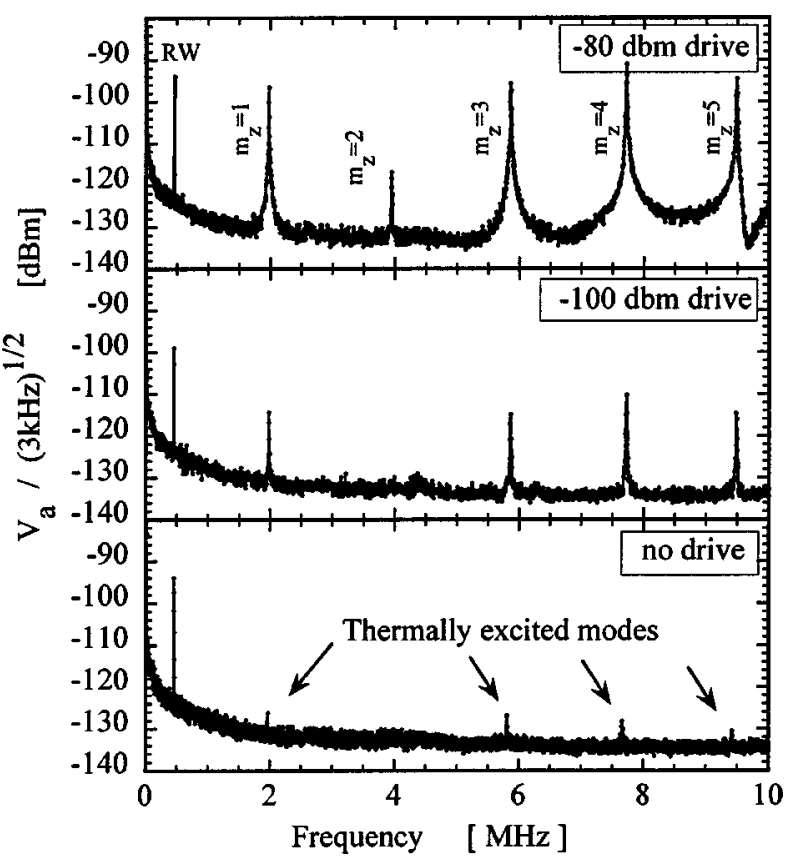

FIG. 2. Spectrum of $m_{\theta}=0, m_{z}=1,2, \ldots, 5$ Trivelpiece-Gould modes for three drive amplitudes including no drive, i.e., thermally excited.

$\equiv(k T / m)^{1 / 2}$ to the wave phase velocity $v_{\phi}$. These modes exhibit exponential damping at a rate $\gamma_{\text {tot }}$ which will be seen to be the sum of $\gamma_{m}$ from inherent plasma mode effects such as Landau damping, and $\gamma_{\ell}$ due to dissipative loading by the receiver.

The antenna/mode coupling is not strongly frequency sensitive, but does show the expected $\sin \left(k_{z} \cdot z\right)$ dependence: the lesser sensitivity for $m_{z}=2$ apparent in Fig. 2 is due to the location and length $L_{a}$ of the detection cylindrical antenna. The peak amplitudes for the continuously driven sinusoidal modes are independent of the bandwidth $(\mathrm{BW}=3$ $\mathrm{kHz}$ ) of the spectrum analyzer; whereas the spectral amplitude of the intermode noise decreases as $(\mathrm{BW})^{-1 / 2}$ as expected. At $V_{\mathrm{exc}}=-100 \mathrm{dBm}$, the mode fluctuations have amplitude $\delta n / n \sim 10^{-5}$. The peak labeled RW is the nonresonant rotating wall drive; the mode measurements presented here have also been obtained with the drive off, and there appears to be no significant coupling between the weak RW drive and the weak TG at incommensurate frequencies.

\section{EMISSION MEASUREMENTS}

Small peaks representing thermally excited modes are still visible in Fig. 2(c) ("no drive") when the transmitter electrode is grounded $\left(V_{\mathrm{exc}}=0\right)$. These peaks have amplitudes on the receiving antenna of $V_{a}=-124 \mathrm{dBm}$ with a receiver bandwidth of $3 \mathrm{kHz}$, representing voltage fluctuations on the electrode with spectral intensity $V_{a} / \sqrt{B W}$ $\approx 2.6 n V / \sqrt{\mathrm{Hz}}$. Here, the mode amplitudes scale as $B W^{-1 / 2}$ as expected for $B W<\gamma$, since the measured power $\left(\propto V_{a}^{2}\right)$ is a fraction $B W / \gamma$ of the total mode power in the antenna circuit.

Figure 3 shows received spectra of the thermally excited $m_{z}=1$ mode for four different plasma temperatures. These analogue analyzer scans over $\Delta f \sim 100 \mathrm{kHz}$ required about

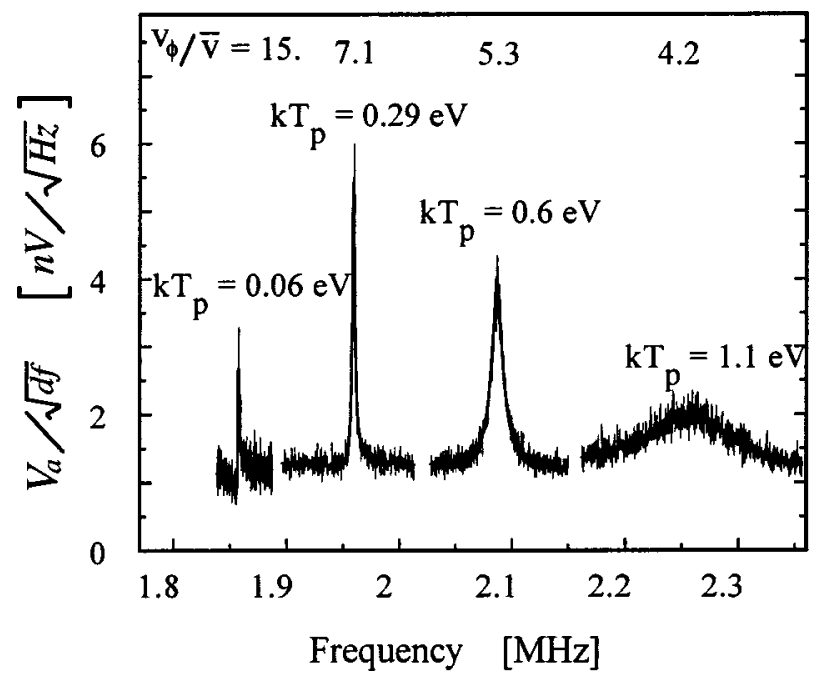

FIG. 3. Spectra of the thermally excited $m_{\theta}=0, m_{z}=1$, mode for different plasma temperatures; the solid lines are fits to Eq. (7). The temperature $T_{p}$ is from emission/reflection measurement.

10 seconds to complete on a steady-state plasma confined with a "rotating wall;" alternately, digital fast Fourier transform (FFT) spectra could be obtained from $0.1 \mathrm{~s}$ of the antenna voltage sampled at $10^{7} \mathrm{samples} / \mathrm{s}$. The mode frequency $f_{m}$ increases slightly with temperature, as expected from Eq. (1). The width of the spectral peak represents the total damping, $\gamma_{\text {tot }}$. This total damping consists of the internal "mode" damping $\gamma_{m}$ and the external "load" damping $\gamma_{\ell}$ :

$$
\gamma_{\mathrm{tot}} \equiv \gamma_{m}+\gamma_{\ell} \text {. }
$$

Here the internal damping is predominantly Landau damping, but any other internal damping (e.g., collisional) is also included in $\gamma_{m}$. The width of the peaks in Fig. 3 increases substantially as Landau damping becomes significant for $k T_{p} \geqslant 0.5 \mathrm{eV}$, i.e., for $v_{\phi} / \bar{v} \lesssim 5$. For the lowest temperature shown in Fig. 3, a substantial distortion of the Lorentzian plasma mode is observed; this will be seen to represent receiver noise reflected by the plasma.

Figure 1(b) shows a circuit modeling the reception of thermal noise from the plasma. Near a plasma mode at frequency $\omega_{m}=2 \pi f_{m}$ with intrinsic damping $\gamma_{m}$, the ratio of antenna current to voltage (i.e., the admittance) $Z_{m}^{-1}$ is given by a simple pole, as

$$
Z_{m}^{-1}=\frac{\mathcal{G} \omega_{m}^{2}}{i\left(\omega-\omega_{m}\right)+\gamma_{m}} .
$$

$\mathcal{G}$ is the geometric (capacitive) coupling coefficient between the plasma mode and the receiving electrode; here $\mathcal{G} \approx 0.5 \mathrm{pF}$ (or $0.5 \mathrm{~cm}$ in CGS units). On resonance, the mode impedance is real with magnitude

$$
R_{m} \equiv Z_{m}^{\mathrm{Re}}\left(\omega_{m}\right)=\gamma_{m} / \mathcal{G} \omega_{m}^{2},
$$

where $Z^{\operatorname{Re}} \equiv \operatorname{Re}\{Z\}$ and $Z^{\operatorname{Im}} \equiv \operatorname{Im}\{Z\}$. The voltage $V_{m}$ represents "white" noise from the plasma particles. Nyquist's theorem predicts $V_{m}=\sqrt{4 k T_{p} \operatorname{Re}\left(Z_{m}\right) d f}$. The currents flow through a resonant mode impedance $Z_{m}$, and then through a load impedance $Z_{\ell}$ with its inevitable white noise. 
The external electronics (load) has measured (input) resistance $R_{\ell}$ and capacitance $C_{\ell}$, or a total load impedance $Z_{\ell}$ given by

$$
Z_{\ell}^{-1}=R_{\ell}^{-1}+i \omega C_{\ell},
$$

which is essentially constant over the mode resonance. The load impedance is easily measured with a vector impedance meter. This almost constant $Z_{\ell}$ differs from the resonantcircuit loads commonly used in harmonic traps with a small number of particles, ${ }^{9}$ simplifying the spectral interpretation somewhat.

Nyquist's theorem says that the spectral density of the square of the noise voltage is proportional to $k T$ times the real part of the impedance, for both the plasma and the load noise sources. A voltage-divider fraction $Z_{\ell} /\left(Z_{m}+Z_{\ell}\right)$ of the thermal plasma-mode voltage $V_{m}$ will be measured on the electrode as $V_{a}$, together with an analogous fraction $Z_{m} /\left(Z_{m}+Z_{\ell}\right)$ of the (uncorrelated) load noise $V_{\ell}$. This gives

$$
\frac{V_{a}^{2}(f)}{d f}=4 k T_{p} Z_{m}^{\mathrm{Re}}\left|\frac{Z_{\ell}}{Z_{m}+Z_{\ell}}\right|^{2}+4 k T_{\ell} \quad Z_{\ell}^{\mathrm{Re}}\left|\frac{Z_{m}}{Z_{m}+Z_{\ell}}\right|^{2} .
$$

The voltage $V_{m}$ from electron thermal motion in the plasma is uncorrelated with the Johnson noise of the load $V_{\ell}$. Using Eqs. (2), (3), and (5), Eq. (6) can be explicitly written as

$$
\begin{aligned}
\frac{V_{a}^{2}(f)}{d f}= & 4 k T_{p} R_{m} \frac{\left|Z_{\ell}\right|^{2}}{\left|R_{m}+Z_{\ell}^{\mathrm{Re}}\right|^{2}} \frac{\gamma_{\mathrm{tot}}^{2}}{\gamma_{\mathrm{tot}}^{2}+\left(\omega-\omega_{m}^{\prime}\right)^{2}} \\
& +4 k T_{\ell} Z_{\ell}^{\mathrm{Re}} \\
& \times\left\{1-\frac{2\left(\omega-\omega_{m}^{\prime}\right) \delta \omega_{m}+\left(\gamma_{\mathrm{tot}}^{2}-\gamma_{m}^{2}-\delta \omega_{m}^{2}\right)}{\gamma_{\mathrm{tot}}^{2}+\left(\omega-\omega_{m}^{\prime}\right)^{2}}\right\},
\end{aligned}
$$

where

$$
\begin{aligned}
& \gamma_{\mathrm{tot}} \equiv \gamma_{m}+\gamma_{\ell}=\left(1+\frac{Z_{\ell}^{\mathrm{Re}}}{R_{m}}\right) \gamma_{m}, \\
& \delta \omega_{m} \equiv \omega_{m}-\omega_{m}^{\prime} \equiv Z_{\ell}^{\mathrm{Im}} \omega_{m}^{2} \mathcal{G} .
\end{aligned}
$$

The first term of Eq. (7) describes the Lorentzian "plasma" emission spectrum centered at $\omega_{m}^{\prime}$ of width $\gamma_{\text {tot }}$, with amplitude proportional to $k T_{p} R_{m}$. Thus, the emission spectrum alone does not determine $k T_{p}$ unless prior knowledge of the coupling coefficient $\mathcal{G}$ allows $R_{m}$ to be obtained from Eq. (4). The second term describes the "load" noise as a uniform background, plus a "dip and peak" from the $(\omega$ $\left.-\omega_{m}^{\prime}\right)$ term, plus a Lorentzian absorption, with all three spectral components proportional to $k T_{\ell} Z_{\ell}^{\mathrm{Re}}$. In practice, making the load about as "hot" as the plasma produces the optimal spectra, allowing a calibrated determination of $k T_{p}$ in one measurement.

Figure 4(a) shows the received spectrum of the $m_{\theta}=0$, $m_{z}=1$ mode in the EV apparatus, when the load is much colder than the plasma. The spectrum is completely described by Eq. (7). Since $Z_{\ell}$ is known, the spectrum is parametrized by the plasma temperature $T_{p}$, mode frequency $\omega_{m}$, mode damping $\gamma_{m}$, coupling coefficient $\mathcal{G}$ and load

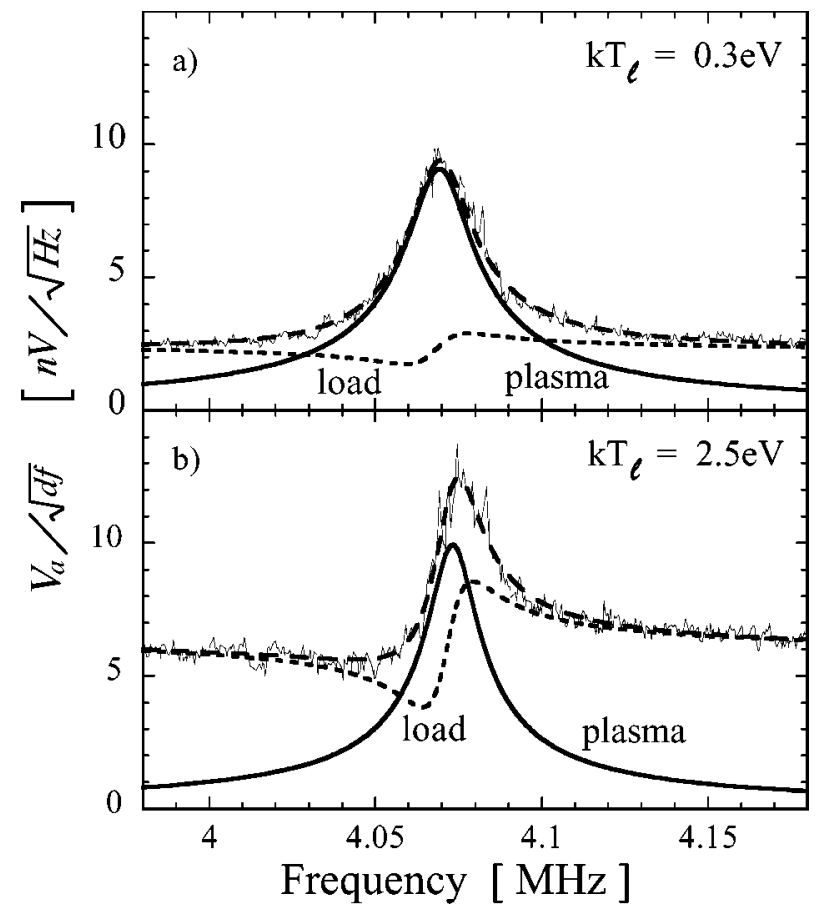

FIG. 4. (a) Spectra of the thermally excited $m_{\theta}=0, m_{z}=1$ mode for $k T_{p}$ $=1.9 \mathrm{eV}$ and $k T_{\ell}=0.3 \mathrm{eV}$. (b) Same with noise added to the receiver $\left(k T_{\ell}=2.5 \mathrm{eV}\right)$. The long-dashed line is Eq. (7) fitted to the data, the solid line is the plasma component, and the short-dashed line is the load noise filtered by the plasma.

temperature $T_{\ell}$. However, the "load" component is too small for the characteristic dip and peak to give an accurate calibration of the coupling coefficient $\mathcal{G}$. Using $\mathcal{G}=0.43 \mathrm{pF}$ (0.39 $\mathrm{cm}$ in CGS) from a separate measurement described below, we obtain $k T_{p}=1.89 \mathrm{eV}, f_{m}=4.063 \mathrm{MHz}, \gamma_{m} / \omega_{m}$ $=2.1 \times 10^{-3}$ and $k T_{\ell}=0.35 \mathrm{eV}$.

In contrast in Fig. 4(b), noise has been deliberately added to the receiver, corresponding to an effectively higher load temperature. The received spectrum has the same Lorentzian "plasma" component, but the dip and peak from the plasma "shorting" the load noise is more pronounced. This phase-sensitive reflection and absorption of the load noise by the plasma determines the antenna coupling coefficient $\mathcal{G}$. A five-parameter fit to the received spectrum of Fig. 4(b) then gives $k T_{p}=1.84 \mathrm{eV}, f_{m}=4.067 \mathrm{MHz}, \gamma_{m} / \omega_{m}$ $=1.6 \times 10^{-3}, \mathcal{G}=0.43 \mathrm{pF}(0.39 \mathrm{~cm}$ in CGS $)$, and $k T_{\ell}=2.5$ $\mathrm{eV}$. The standard dump diagnostic gives $k T_{p}^{\mathrm{dump}}=1.9 \mathrm{eV}$, with no measurable difference for Figs. 4(a) and 4(b).

Figure 4(b) demonstrates that the plasma temperature can be obtained in one measurement if the load is "noisy enough." Of course, if the emission from the load were to dominate the spectrum, the plasma component proportional to $T_{p}$ might be obscured. For Eq. (6) to be valid, the load must be uncorrelated with the plasma mode. Using a swept analogue analyzer, a sine wave of constant amplitude which tracks the frequency of the receiver (from a tracking generator) satisfies the criterion. When digitizing the wave form for FFT analysis, a "random" load current should be added to the antenna/receiver junction. The damping rate $\gamma_{m}$ is mea- 


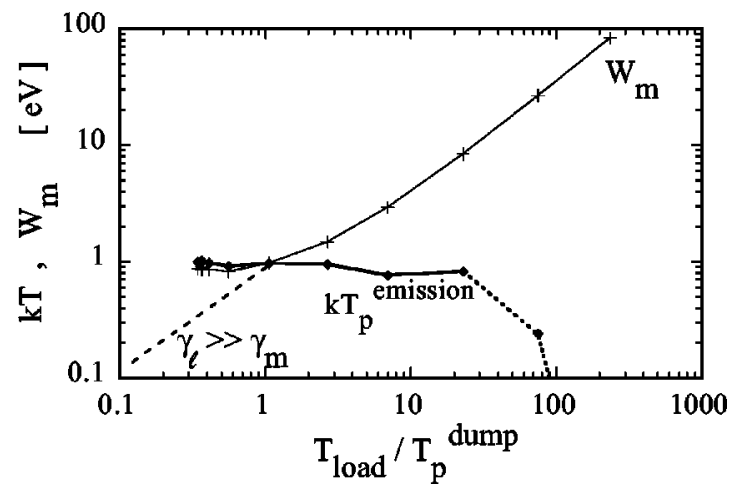

FIG. 5. Plasma temperature $T_{p}$ as obtained by emission and average mode energy $W_{m}$ obtained from Eq. (9) for a plasma temperature of $0.92 \mathrm{eV}$ versus normalized load temperature.

surably smaller when the noise added to the load drives the mode to large amplitude, because the wave traps particles at the phase velocity, thereby reducing the Landau damping. The thermal component [solid curve in Fig. 4(b)] can be viewed as a small test wave in the presence of a larger amplitude wave excited by the load noise.

To establish the load "temperature" range over which the five-parameter fit determines $T_{p}$ correctly, we varied $T_{\ell}$ over the range $0.35<T_{\ell} / T_{p}<200$ with a fixed $k T_{p}=0.9 \mathrm{eV}$. Figure 5 indicates that $k T_{p}$ is obtained satisfactorily in one measurement if $T_{\ell} / T_{p} \leqslant 20$.

Thermally excited modes are in equilibrium with both the plasma at temperature $T_{p}$ and the load at temperature $T_{\ell}$. Theory suggests that the plasma mode reaches an equilibrium average energy $W_{m}$ given by

$$
0=\frac{d}{d t} W_{m}=\gamma_{m}\left(k T_{p}-W_{m}\right)+\gamma_{\ell}\left(k T_{\ell}-W_{m}\right) .
$$

If the mode were subject to other energy couplings, these couplings would also appear on the right-hand side of Eq. (8). The average equilibrium mode energy, given by

$$
W_{m}=\frac{\gamma_{m} k T_{p}+\gamma_{\ell} k T_{\ell}}{\gamma_{m}+\gamma_{\ell}},
$$

increases as $T_{\ell} / T_{p}$ increases, i.e., the density fluctuations associated with the mode are increasing in the plasma. Clearly the mode is in thermal equilibrium with the plasma and with the load. Therefore a high temperature load increases the average energy of the mode $W_{m}$; conversely a well coupled (i.e., large $\gamma_{\ell}$ ) cold load will reduce $W_{m}$. Note that the plasma temperature $T_{p}$ (i.e., the temperature of the particles) remains unchanged because one mode carries $\frac{1}{2} k T$ of electrostatic energy while the plasma has $N_{\text {tot }} k T$ of energy where $N_{\text {tot }} \simeq 10^{9}$ electrons.

Similarly, a feedback circuit can present a "negative impedance" coupling to the mode. We have actively damped thermally excited modes, reducing $W_{m}$ by a factor of 25 , and observe no measurable change in $T_{p}$.

\section{THE GEOMETRICAL COUPLING COEFFICIENT}

One can alternately calculate the geometrical coupling coefficient $\mathcal{G}$, or determine it with a separate reflection/ absorption measurement. As we have seen, the emission spectrum with a noisy receiver effectively incorporates a reflection/absorption measurement.

We calculate the coupling coefficient $\mathcal{G}$ analytically using kinetic theory. Analysis of a uniform density collisionless plasma of radius $r_{p}$ with $z$-periodic boundaries of period $L_{p}$ reproduces the impedance of Eq. (3) for frequencies near a plasma resonance. In the limit of $T \rightarrow 0$, assuming that $\lambda_{D}$ $\ll r_{p}$ and that $k_{z} r_{w} \ll 1$, we find that

$$
\mathcal{G}=\frac{4 \pi \varepsilon_{0} L_{p} F_{m}^{2}}{1+x^{2} \ln ^{2}\left(r_{\omega} / r_{p}\right)} .
$$

Here $\quad F_{m} \equiv\left(m_{z} \pi\right)^{-1}\left[\sin \left(m_{z} \pi z_{2} / L_{p}\right)-\sin \left(m_{z} \pi z_{1} / L_{p}\right)\right]$, with $z_{1}$ and $z_{2}$ representing the left and right ends of the antenna cylinder; $x$ is a dimensionless quantity that satisfies the equation $x J_{1}(x) \ln \left(r_{w} / r_{p}\right)=J_{0}(x)$, and is related to the frequency of the plasma mode by $x=k_{z} r_{p}\left(\omega_{p}^{2} / \omega_{m}^{2}-1\right)^{1 / 2}$. For $r_{\omega} / r_{p} \gg 1$, one sees $x \approx \sqrt{2 / \ln \left(r_{w} / r_{p}\right)}$, which implies $\mathcal{G}$ $\simeq 4 \pi \varepsilon_{0} L_{p} F_{m}^{2} /\left[1+2 \ln \left(r_{w} / r_{p}\right)\right]$. All equations except Eq. (10) are valid in CGS or SI; in CGS Eq. (10) would have no $4 \pi \varepsilon_{0}$.

Equation (10) presumes that the plasma column is penetrating the cylindrical antenna completely. When the antenna is at the end of the plasma and the plasma only partially penetrates the electrode, the plasma end point $z_{p}$ would replace the electrode end $z_{2}$ in estimating $F_{m}$. A more complete kinetic analysis of a Maxwellian distribution of "fully dressed" test particles including the plasma dielectric properties $^{22}$ reproduces the Nyquist theorem of Eq. (7), and also gives the nonresonant Debye-shielded fluctuations. For example, each (axial) half of the plasma has total (frequencyintegrated) nonresonant fluctuations $(\delta N)^{2}=(\delta q)^{2} / e^{2}$ $\sim \mathcal{O}(0.1)\left(\lambda_{D} / r_{p}\right)^{3} N$ for $\lambda_{D} \leq r_{p}$, showing a strong reduction below the $(\delta N)^{2} \propto N$ fluctuations expected for fully uncorrelated particles. ${ }^{23}$

A completely experimental determination of $\mathcal{G}$ can be obtained with a reflection/absorption measurement, ${ }^{10}$ as shown in Fig. 6(a). The direct measurement of $Z_{m}$ uses a directional coupler and lock-in detector to determine the complex reflection coefficient $r(f)$ for a weak wave at frequency $f$ incident on the receiving antenna and plasma. This reflection coefficient is defined as the voltage fraction (and phase) which is reflected by the plasma-loaded antenna compared to that reflected by an open circuit without antenna or plasma, i.e.,

$$
r \equiv \frac{V_{\text {refl }}(\text { plasma })}{V_{\text {refl }}(\text { open })} .
$$

This reflection coefficient depends on the impedance $Z_{\text {tot }}$ connected to the directional coupler compared to the impedance $Z_{0}=50 \Omega$ of the coupler itself, and is given implicitly by

$$
Z_{\mathrm{tot}}=Z_{0} \frac{(1+r)}{(1-r)} .
$$




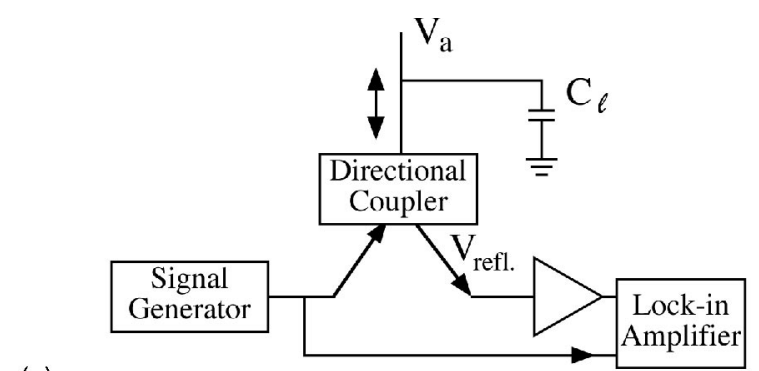

(a)

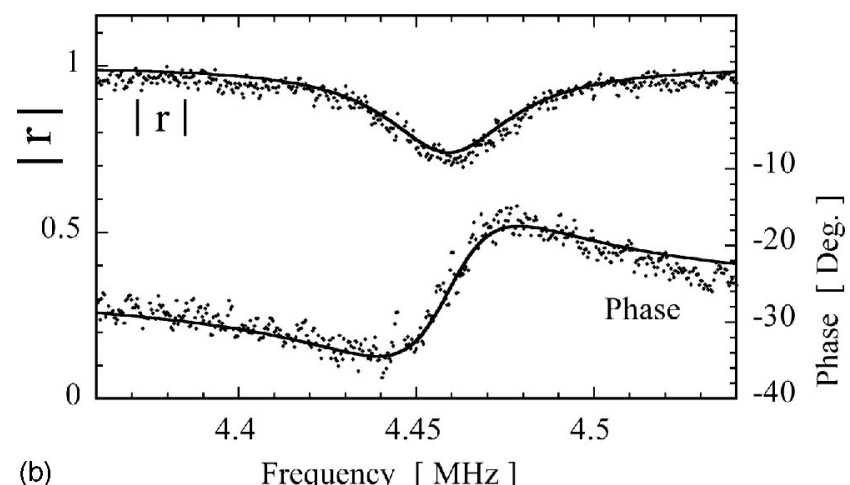

(b)

Frequency [ $\mathrm{MHz}$ ]

FIG. 6. (a) Reflection/absorption electronics. (b) Measured magnitude and phase of the reflection coefficient $r$.

Here, $Z_{\mathrm{tot}}^{-1}=Z_{m}^{-1}+i \omega C_{\ell}$ is the total impedance of the plasma-loaded antenna, given by the plasma impedance $Z_{m}$ of Eq. (3) in parallel with the capacitance $C_{\ell}$ of the electrode and connecting cable.

Figure 6(b) shows an example of the measured amplitude and phase of the reflected wave as the frequency is scanned across the $m_{z}=1$ mode. The entire signal is reflected at frequencies $f$ far from the mode frequency $f_{m}$, because the plasma impedance [Eq. (3)] is large off-resonance, i.e., $\left|Z_{m}(f)\right| \gg Z_{0}$. On resonance, about $25 \%$ of the incident wave is absorbed by the plasma, and $75 \%$ is reflected. In essence, the depth of the absorption dip indicates how close $R_{m}$ is to the $50 \Omega$ of the directional coupler, since $Z_{m}^{\operatorname{Im}}\left(f_{m}\right)=0$. A fit (solid line) to the $r(f)$ data using Eqs. (3) and (12) gives the parameters of $Z_{\text {tot }}$ as $R_{m}=329 \Omega\left(=366 \times 10^{-12} \mathrm{~s} / \mathrm{cm}\right.$ in CGS), $f_{m}=4.458 \mathrm{MHz}, \gamma_{m} / \omega_{0}=4.6 \times 10^{-3}$, and $C_{\ell}=158$ $\mathrm{pF}$, resulting in $\mathcal{G}=\gamma /\left(\omega^{2} R\right)=0.49 \mathrm{pF}$ (or $0.44 \mathrm{~cm}$ in CGS).

Figure 7 shows the coupling coefficient $\mathcal{G}$ is robust, i.e., it changes by less than a factor of 2 when the plasma temperature changes from $0.7 \mathrm{eV}$ to $3.5 \mathrm{eV}$. The simple low temperature limit of Eq. (10), for EV plasma parameters, gives $\mathcal{G}=0.42 \mathrm{pF}$, shown with a dashed line. In contrast, the mode impedance $R_{m}$ varies from $30 \Omega$ to $2000 \Omega$ in that temperature range, as Landau damping increases the mode damping $\gamma_{m}$. Since $\mathcal{G}$ is almost constant, Eq. (4) predicts that $R_{m}$ will increase as $\gamma_{m} / \omega_{m}^{2}$. The dashed line represents $R_{m}$ $=\gamma_{\text {Landau }} / \omega_{m}^{2} \mathcal{G}$, where $\gamma_{\text {Landau }}$ is calculated from plasma parameters. The small discrepancies may be due to a $20 \%$ error in the temperature calibration of the EV apparatus, or possibly finite length plasmas may require a correction to Landau damping. Figure 8 illustrates that $R_{m}$ is directly related to the mode damping $\gamma_{m} / \omega_{m}$; here again the dashed line is the simple theory prediction from $\mathcal{G}=0.42 \mathrm{pF}$.

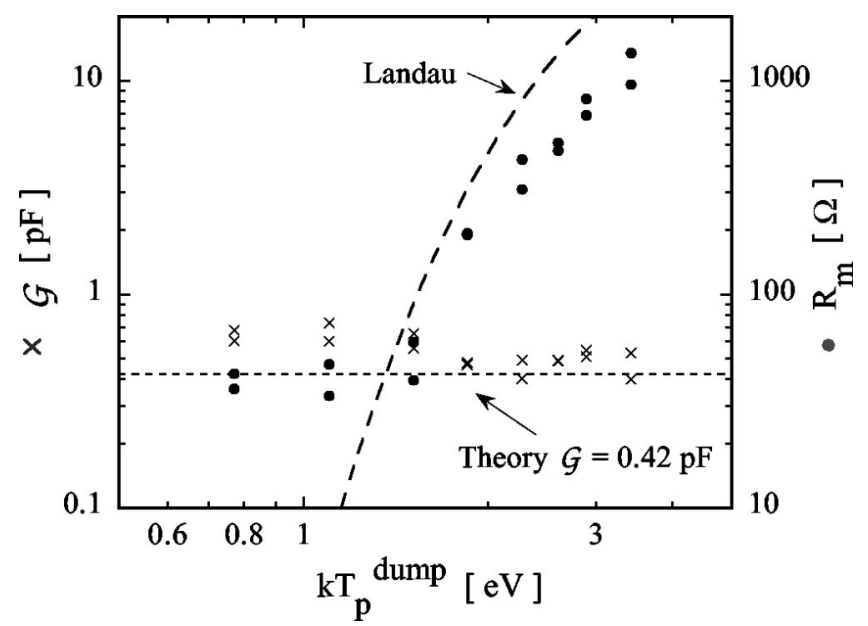

FIG. 7. Coupling coefficient $\mathcal{G}$ and plasma-mode impedance $R_{m}$ versus plasma temperature.

\section{TEMPERATURE DIAGNOSTIC}

Figure 9 displays the plasma temperature $T_{p}^{\text {emission }}$ obtained from the emission spectra versus the plasma temperature $T_{d}^{\text {dump }}$ measured by dumping the plasma. Data were taken for plasmas with a range of "geometric" parameters $\left(n, r_{p}, L_{p}\right)$ on both EV (circles) and IV (triangles), with varied amounts of plasma heating. Most of the values of $T_{p}^{\text {emission }}$ were obtained from four-parameter fits to the emission spectra, together with a separate measurement of $\mathcal{G}$ for each $\left(n, r_{p}, L\right)$.

On IV, the value of $\mathcal{G}$ was determined from a single five-parameter fit to the non-Lorentzian " $k T=0.06 \mathrm{eV}$ " spectrum of Fig. 3, giving $\mathcal{G}=0.21 \mathrm{pF}(0.19 \mathrm{~cm}$ in CGS $)$. Implementation of the "high temperature load" technique has obviated the directional coupler reflection measurements, and essentially identical values of $T_{p}$ are obtained with a single spectrum.

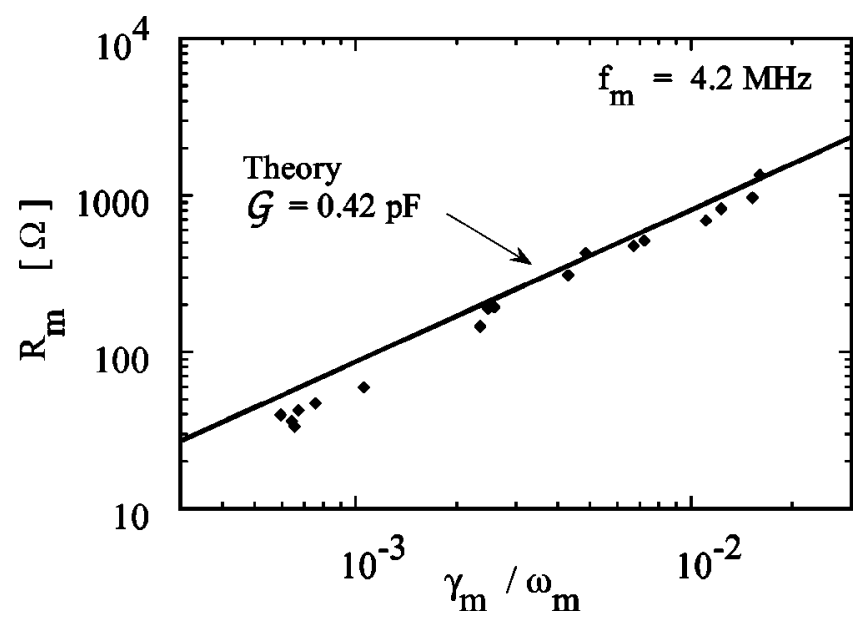

FIG. 8. Normalized plasma mode impedance versus the plasma-mode damping rate. 


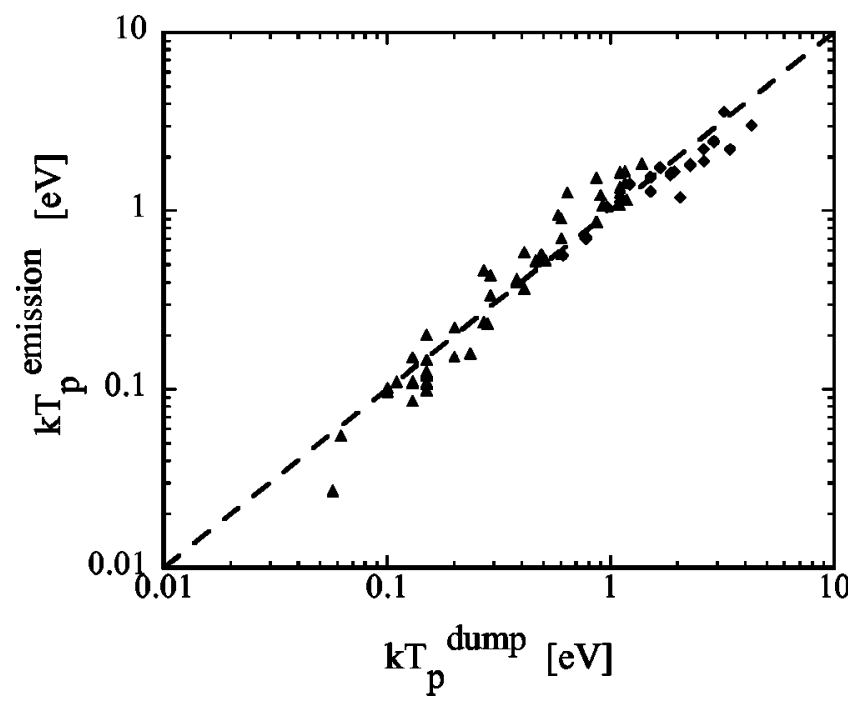

FIG. 9. Plasma temperature measured by emission technique, compared to the standard dump temperature measurement. The triangles are from the IV apparatus and the diamonds are from the EV apparatus.

\section{DISCUSSION}

It should be emphasized that steady-state plasmas often exhibit spectral peaks which are much larger than thermal, as shown in Figs. 2(a) and 2(b), because the mode is being externally driven. More subtly, noise on the ostensibly steady confinement voltage $V_{\text {conf }}$ or other rf signals may stimulate particular plasma modes without proportionately increasing the plasma temperature. In practice, the standard (destructive) dump diagnostic is valuable in establishing that the apparatus is "quiet," and that the mode couples only to $T_{p}$ and $T_{\ell}$. Note that under "noisy" confining voltage conditions, $T_{p}^{\text {emission }}$ does not scale linearly with $T_{p}^{\text {dump }}$.

After carefully eliminating unwanted external damping, the measured total mode damping $\gamma_{\text {tot }}$ is consistent with the theoretical perspective of inherent plasma mode damping $\gamma_{m}$ and the receiver load-induced damping $\gamma_{\ell}$ calculated from the known $Z_{\ell}^{\mathrm{Re}}$. The analysis presumes that $\gamma_{\mathrm{tot}}=\gamma_{m}+\gamma_{\ell}$; any unforeseen external damping would be included erroneously into $\gamma_{m}$. The smallest mode damping obtained on EV in regimes where Landau damping should be negligibly small is $\gamma_{m} / \omega_{m} \simeq 7 \times 10^{-4}$; presuming this residual damping arises from unknown couplings to external resistances, Eq. (4) sets a limit to the "unknown" coupling coefficient and impedance connected to $\mathcal{G}_{u} R_{u}<28[\mathrm{pF} \cdot \Omega]$. This represents all electrodes other than the antenna/receiver circuit.

In summary, we observe thermally excited plasma modes capacitively coupled to an antenna with a room temperature amplifier. Using a generalized version of Nyquist's theorem, we have shown that one single nondestructive measurement can determine the coupling coefficient $\mathcal{G}$, the plasma temperature $T_{p}$ and also the load temperature $T_{\ell}$, damping rate $\gamma_{m}$ and mode frequency $\omega_{m} / 2 \pi$. This single measurement technique is possible because the frequency dependences of $Z_{m}(\omega)$ and $Z_{\ell}(\omega)$ are distinguishable. The thermally excited plasma modes are coupled to the theorist's thermal bath ${ }^{6}$ only at rate $\gamma_{m}$, which depends sensitively on temperature; and they are also coupled to the load at temperature $T_{\ell}$ at a rate $\gamma_{\ell}$, which depends on the antenna coupling and receiver parameters. This new technique is now a working diagnostic that may be useful for "expensive" particles as, for example, antimatter.

\section{ACKNOWLEDGMENTS}

We thank Dr. R. E. Pollock for his seminal contributions and Dr. T. M. O’Neil for many fruitful discussions.

This work is supported by Office of Naval Research Grant No. N00014-96-1-0239 and National Science Foundation Grant No. PHY-9876999.

${ }^{1}$ R.G. Greaves and C.M. Surko, Phys. Plasmas 4, 1528 (1997).

${ }^{2}$ M. Amoretti, C. Amsler, G. Bonomi et al., Nature (London) 419, 456 (2002); G. Gabrielse, N.S. Bowden, P. Oxley et al., Phys. Rev. Lett. 89, 213401 (2002)

${ }^{3}$ J.P. Sullivan, S.J. Gilbert, and C.M. Surko, Phys. Rev. Lett. 86, 1494 (2001).

${ }^{4}$ K.H. Knoll, G. Marx, K. Hubner, F. Schweikert, S. Stahl, C. Weber, and G. Werth, Phys. Rev. A 54, 1199 (1996).

${ }^{5}$ T.B. Mitchell, J.J. Bollinger, D.H.E. Dubin, X.-P. Huang, W.M. Itano, and R.H. Baughman, Science 282, 1290 (1998).

${ }^{6}$ T.M. O'Neil and D.H.E. Dubin, Phys. Plasmas 5, 2163 (1998).

${ }^{7}$ D.H.E. Dubin, Phys. Plasmas 5, 1688 (1998); F. Anderegg, X.-P. Huang, C.F. Driscoll, E.M. Hollmann, T.M. O'Neil, and D.H.E. Dubin, Phys. Rev. Lett. 78, 2128 (1997); D.H.E. Dubin, ibid. 79, 2678 (1997); E.M. Hollmann, F. Anderegg, and C.F. Driscoll, ibid. 82, 4839 (1999).

${ }^{8}$ S.A. Prasad and T.M. O'Neil, Phys. Fluids 26, 665 (1983); A.W. Trivelpiece and R.W. Gould, J. Appl. Phys. 30, 1784 (1959).

${ }^{9}$ D.J. Wineland and H.G. Dehmelt, J. Appl. Phys. 46, 919 (1975).

${ }^{10}$ R.W. Gould, Phys. Plasmas 2, 2151 (1995).

${ }^{11}$ I. Fidone, G. Giruzzi, and G. Taylor, Phys. Plasmas 3, 2331 (1996).

${ }^{12}$ R.L. Stenzel and R.W. Gould, J. Appl. Phys. 42, 4225 (1971).

${ }^{13}$ N. Meyer-Vernet and C. Perche, J. Geophys. Res., [Atmos.] 94, 2405 (1989).

${ }^{14}$ S. Nunomura, J. Goree, S. Hu, X. Wang, and A. Bhattacharjee, Bull. Am. Phys. Soc. 46, 22 (2001).

${ }^{15}$ A. Gillespie and F. Raab, Phys. Rev. D 52, 577 (1995).

${ }^{16}$ K.L. Bowles, G.R. Ochs and J.L. Green, J. Res. Natl. Bur. Stand., Sect. D 66D, 395 (1962).

${ }^{17}$ N. Rostoker and M.N. Rosenbluth, Phys. Fluids 3, 1 (1960).

${ }^{18}$ F. Anderegg, E.M. Hollmann, and C.F. Driscoll, Phys. Rev. Lett. 81, 4875 (1998); E.M. Hollmann, F. Anderegg, and C.F. Driscoll, Phys. Plasmas 7, 2776 (2000)

${ }^{19}$ D.L. Eggleston, C.F. Driscoll, B.R. Beck, A.W. Hyatt, and J.H. Malmberg, Phys. Fluids B 4, 3432 (1992).

${ }^{20}$ B.R. Beck, J. Fajans, and J.H. Malmberg, Phys. Plasmas 3, 1250 (1996).

${ }^{21}$ R.C. Davidson, Physics of Nonneutral Plasmas (Addison-Wesley, Reading, MA, 1990), Sec. 5.5.2.

${ }^{22}$ N. Krall and A.W. Trivelpiece, Principles of Plasma Physics (McGrawHill, New York, 1973), Chap. 11.

${ }^{23}$ N.T. Nakata, G.W. Hart, and B.G. Peterson, in Non-Neutral Plasma Physics IV, edited by F. Anderegg, L. Schweikhard, and C.F. Driscoll (American Institute of Physics, New York, 2002). 